\title{
Airway structural cells regulate TLR5-mediated mucosal adjuvant activity
}

L Van Maele ${ }^{1,2,3,4,14}$, D Fougeron ${ }^{1,2,3,4,14}$, L Janot ${ }^{5,6,14}$, A Didierlaurent ${ }^{7}$, D Cayet ${ }^{1,2,3,4}$, J Tabareau ${ }^{1,2,3,4}$, M Rumbo $^{8}$, S Corvo-Chamaillard ${ }^{1,2,3,4}$, S Boulenouar ${ }^{1,2,3,4}$, S Jeffs $^{9}$, L Vande Walle ${ }^{10,11}$, M Lamkanfi ${ }^{10,11}$,

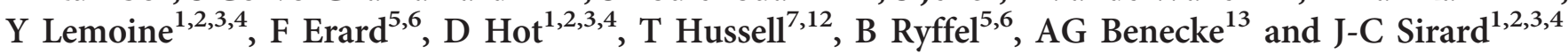

Antigen-presenting cell (APC) activation is enhanced by vaccine adjuvants. Most vaccines are based on the assumption that adjuvant activity of Toll-like receptor (TLR) agonists depends on direct, functional activation of APCs. Here, we sought to establish whether TLR stimulation in non-hematopoietic cells contributes to flagellin's mucosal adjuvant activity. Nasal administration of flagellin enhanced T-cell-mediated immunity, and systemic and secretory antibody responses to coadministered antigens in a TLR5-dependent manner. Mucosal adjuvant activity was not affected by either abrogation of TLR5 signaling in hematopoietic cells or the presence of flagellin-specific, circulating neutralizing antibodies. We found that flagellin is rapidly degraded in conducting airways, does not translocate into lung parenchyma and stimulates an early immune response, suggesting that TLR5 signaling is regionalized. The flagellin-specific early response of lung was regulated by radioresistant cells expressing TLR5 (particularly the airway epithelial cells). Flagellin stimulated the epithelial production of a small set of mediators that included the chemokine CCL20, which is known to promote APC recruitment in mucosal tissues. Our data suggest that (i) the adjuvant activity of TLR agonists in mucosal vaccination may require TLR stimulation of structural cells and (ii) harnessing the effect of adjuvants on epithelial cells can improve mucosal vaccines.

\section{INTRODUCTION}

Vaccine formulation requires adjuvants to increase the immune response to antigen. A fundamental question in vaccine research is how immunity is stimulated by adjuvants. The most commonly used adjuvants for human vaccination by intramuscular and subcutaneous administrations are aluminum salts or alum. The mechanism of action of alum has been associated both with depot effects that facilitate antigen delivery to the immune system and to danger signaling through innate immune receptors like the nucleotide oligomerization domainlike receptor (NLR) family pyrin domain containing 3 (NLRP3) inflammasome or DNA sensors. ${ }^{1,2}$ In the past decade, novel adjuvants that specifically target Toll-like receptors
(TLRs) or NLRs have been characterized. ${ }^{3,4}$ For example, lipopolysaccharide (LPS), flagellin, and unmethylated CpG DNA (CpG) that are sensed by TLR4, TLR5, and TLR9, respectively, trigger the production of various cytokines and chemokines. ${ }^{5}$ Thus, TLR signaling that is instrumental for the induction of innate immunity and the concomitant ignition of adaptive immune responses, activates several types of immune cells or non-immune cells like epithelial cells. ${ }^{6}$ Antigenpresenting cells (APCs) like dendritic cells (DCs) are main responders to TLR agonists. ${ }^{7}$ The actual dogma specifies that TLR signaling promotes functional maturation of DCs resulting in antigen presentation to naive $\mathrm{T}$ and $\mathrm{B}$ lymphocytes. ${ }^{8}$ Most vaccine candidates or licensed vaccines containing

\footnotetext{
${ }^{1}$ Institut Pasteur de Lille, Centre d'Infection et d'Immunité de Lille, Lille, France. ${ }^{2}$ Institut National de la Santé et de la Recherche Médicale, Lille, France. ${ }^{3}$ Centre National de la Recherche Scientifique, Lille, France. ${ }^{4}$ Univ Lille Nord de France, Lille, France. ${ }^{5}$ University of Orléans, Orléans, France. ${ }^{6} \mathrm{CNRS}$ Molecular Immunology and Embryology UMR 6218, Institut de Transgenose, Orléans, France. ${ }^{7}$ Imperial College of London, National Heart and Lung Institute, London, UK. ${ }^{8}$ Universidad Nacional de La Plata, Laboratorio de Investigaciones en el Sistema Inmune, Facultad de Ciencias Exactas, La Plata, Argentina. ${ }^{9}$ Jefferiss Trust Research Laboratories, Imperial College, London, UK. ${ }^{10}$ Department of Medical Protein Research, VIB, Ghent, Belgium. ${ }^{11}$ Department of Biochemistry, Ghent University, Ghent, Belgium. ${ }^{12}$ Manchester Collaborative Centre for Inflammation Research, University of Manchester, Manchester, UK and ${ }^{13}$ Institut des Hautes Études Scientifiques and Centre National de la Recherche Scientifique, Bures-sur-Yvette, France. Correspondence: J-C Sirard (jean-claude.sirard@inserm.fr)

${ }^{14}$ These authors contributed equally to this work.
} 
TLR-based adjuvants (like the monophosphoryl lipid A) are assumed to depend on direct APC activation. ${ }^{1}$

Most life-threatening pathogens target the mucosal barriers, and hosts need to rapidly circumvent their dissemination by regionalized immunity. ${ }^{9}$ Compared with conventional vaccines that are delivered by intramuscular or subcutaneous routes, mucosal vaccines specifically promote regionalized responses. ${ }^{10}$ Thus, mucosal vaccines prevent infections in an early phase through the luminal secretion of sIgA and local T-cell responses. ${ }^{10}$ Mucosal routes of administration require live vaccines or specific adjuvants, i.e., mucosal adjuvants, that are able to alter/breach the epithelial barrier and promote immunomodulation such as enterotoxins and detoxified derivatives from the cholera toxin family. ${ }^{10}$ TLR agonists have shown potency and safety as candidate mucosal adjuvants mainly when formulated in particles. ${ }^{11}$ The mode of action of mucosal adjuvants, including TLR agonists, is far from being understood. Identification of TLR-dependent mechanisms by which mucosal adjuvants increase adaptive immunity is therefore essential for the development of novel vaccine strategies.

Some bacterial flagellins (the major component of flagella of many mucosal pathogens) are unique agonists for TLR5 and NLR family CARD domain-containing protein 4 (NLRC4)dependent signaling. ${ }^{12,13}$ Flagellins are effective adjuvants for T-cell-mediated and antibody responses by systemic routes of immunization in a process dependent on TLR5 and/or NLRC4 inflammasome. ${ }^{14-18}$ Interestingly, mucosal administration of flagellin with antigen stimulates strong antigen-specific adaptive immune responses, especially secretory IgG and IgA, and serum antibodies. ${ }^{18-20}$ Most studies correlated the mucosal adjuvant capacity of flagellin to signaling in DCs from the intestinal or respiratory tracts. ${ }^{12,15,21,22}$ For instance, DC stimulation by flagellin activates processing, presentation, co-stimulatory function, and secretion of interleukin (IL)12, IL-23, and/or IL-6, thereby promoting activation of Th1, Th2, and Th17 cell-, antibody-mediated immunity as well as innate lymphoid cells. ${ }^{15,16,22-24}$ On the other hand, flagellin is a potent stimulus for epithelial cells. ${ }^{12,25,26}$ In the mucosal context, the epithelial cells are the first sentinel cells to be exposed to microbes and are involved in the immediate triggering of innate immunity by sensing through TLRs and related innate receptors. ${ }^{27} \mathrm{We}$ and others recently observed that, after nasal administration of flagellin, TLR5 signaling in radioresistant lung cells is sufficient to trigger compartmentalized mucosal innate immunity, i.e., synthesis of CXCL cytokines and recruitment of neutrophils. ${ }^{28}$ However, whether epithelial cells are involved in the ignition of flagellin-mediated adaptive immune responses after mucosal administration remains to be investigated.

Here we addressed whether mucosal adaptive immunity depends on TLR5-mediated epithelial signaling. Using Tlr $5^{-1-}$ chimera, we showed that adaptive immunity to nasal administration of flagellin requires TLR5-expressing structural cells. Indeed, TLR5 expression in the hematopoietic compartment was not associated to any significant induction of mucosal response. Bronchial and alveolar epithelial cells recapitulated the transcriptional signature of lung response to flagellin. Our results suggest that TLR5-dependent stimulation of epithelium is the driving force for adjuvant activity of flagellin in the mucosa. These findings provide further perspectives in the development of new mucosal adjuvants.

\section{RESULTS}

\section{Mucosal TLR5 signaling stimulates local and systemic adaptive responses}

The TLR5 agonist flagellin or its non-antigenic derivative FliC $_{\triangle 174-400}$ is an efficient adjuvant for vaccination when delivered by the respiratory route. ${ }^{18,32}$ We studied the antibody and $\mathrm{T}$-cell response stimulated via the intranasal (i.n.) route by flagellin formulated with various antigens including ovalbumin (OVA), diphtheria and tetanus toxoids (DT and TT, respectively), keyhole limpet hemocyanin, and the HIV-derived recombinant GP140 protein. We found that flagellin, like cholera toxin, the gold standard mucosal adjuvant, potentiated the systemic and local antibody response in mice to coadministered antigens (Figure 1 and Supplementary Figure 1 online). In serum and bronchoalveolar lavages (BALs) of animals, levels of antigen-specific total IgG and IgG1 were increased, whereas IgG2a or IgG2c production was unchanged (Figures 1a and c). The levels of antigen-specific IgA in BAL were also increased (Figure 1b). To examine the T-cell
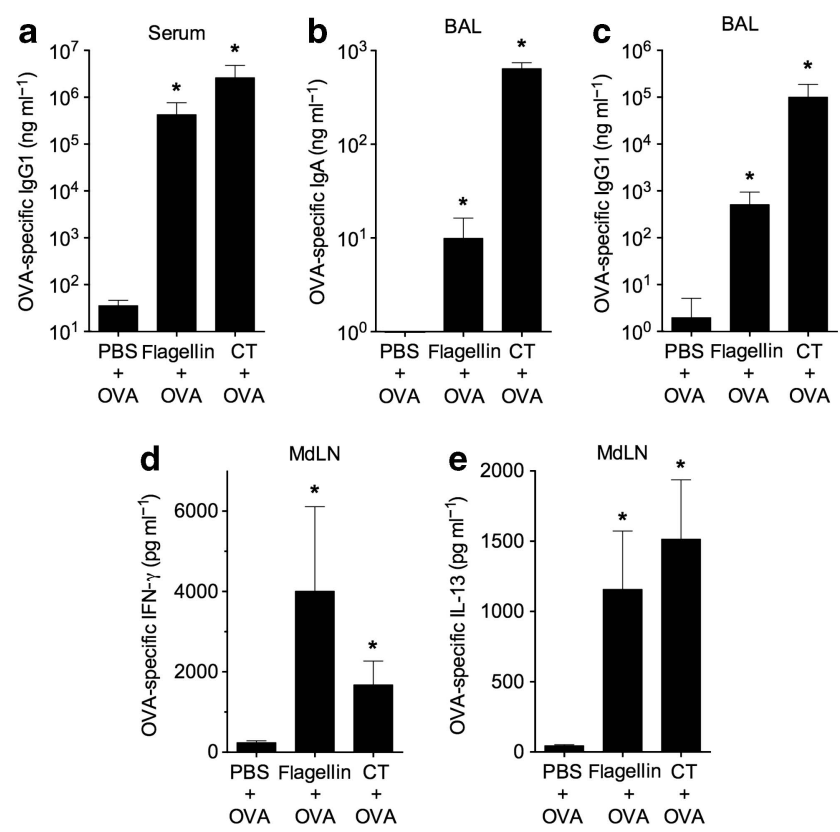

Figure 1 Nasal administration of flagellin stimulates mucosal and systemic adaptive immunity. C57BL/6 mice $(n=6-8)$ were immunized intranasally at days 1 and 21 with ovalbumin (OVA) in phosphate-buffered saline (PBS) \pm flagellin or cholera toxin (CT). (a-c) OVA-specific antibody response. Serum (a) and bronchoalveolar lavage $(B A L)(b, c)$ were collected at day 35 and levels of OVA-specific IgG1 or IgA were determined by ELISA. (d, e) Antigen-specific T-cell response. Mediastinal lymph node (MdLN) cells were collected at day 10 and stimulated $72 \mathrm{~h}$ with OVA, and cytokine levels in supernatant were determined by ELISA. Results are expressed as mean \pm s.d. and are representative of 3-6 experiments. Statistical significance $\left({ }^{\star} P<0.05\right)$ was assessed by Mann-Whitney test compared with the PBS group. 

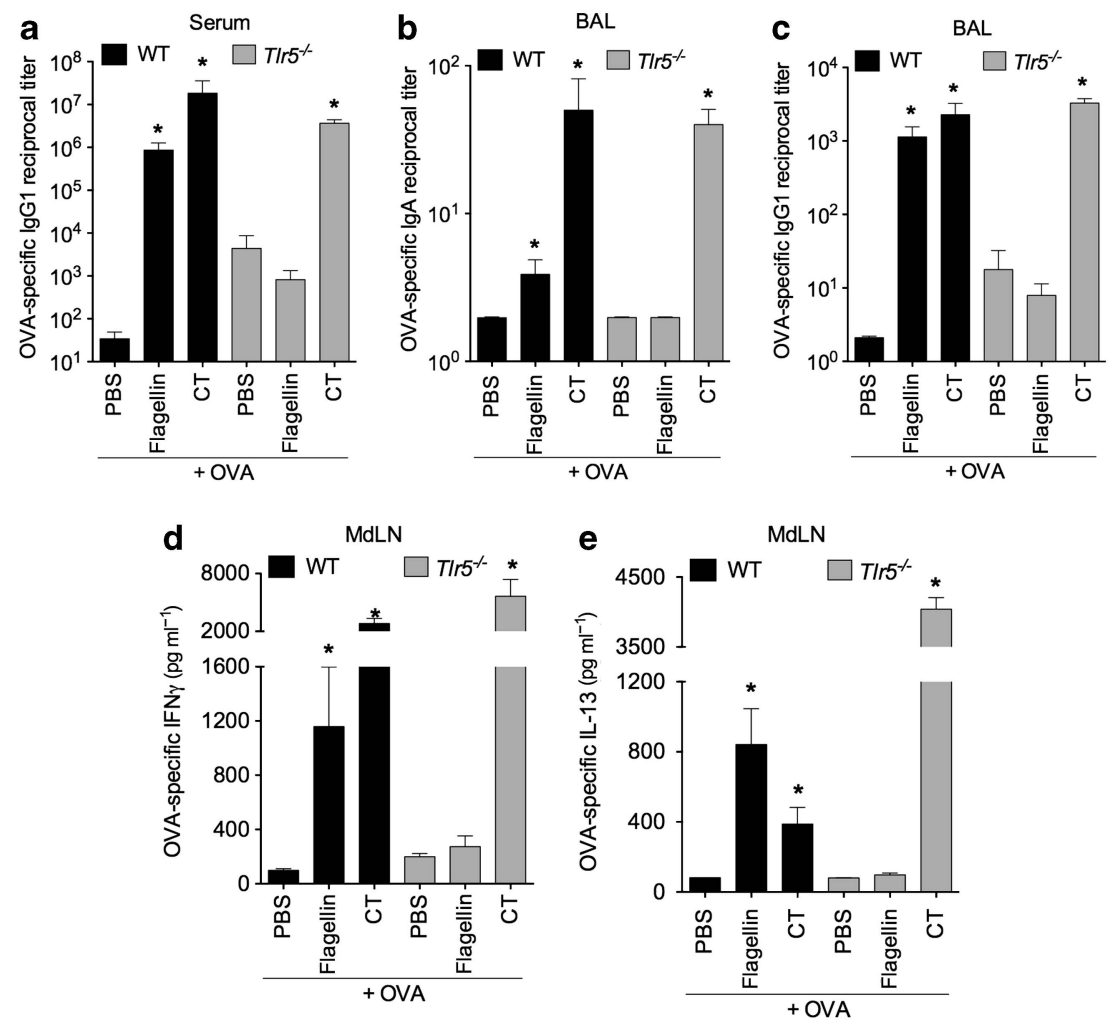

Figure 2 Adjuvant effect of flagellin is TLR5 dependent. C57BL/6 and T/r5 ${ }^{-/-}$mice $(n=4-14)$ were immunized intranasally at days 1 and 21 with ovalbumin (OVA) in phosphate-buffered saline (PBS) \pm flagellin or cholera toxin (CT). Serum, bronchoalveolar lavage (BAL), and mediastinal lymph node (MdLN) were collected at day 35. (a, b) OVA-specific antibody response. Levels of OVA-specific IgG1 or IgA were determined by ELISA in serum (a) and bronchoalveolar lavages (b, c). (d, e) OVA-specific T-cell response. Lymph node cells were stimulated $72 \mathrm{~h}$ with OVA and cytokine levels in supernatant were determined by ELISA. Results are expressed as mean \pm s.e.m. and are representative of 2 experiments. Statistical significance $\left({ }^{\star} P<0.05\right)$ was assessed by Mann-Whitney test compared to the PBS group for each mouse strain.

response, cells from mediastinal lymph nodes (MdLNs) and spleens of animals immunized with OVA-containing vaccines were activated ex vivo (Figures 1d and e and data not shown). In agreement with the antibody patterns, the OVA-specific response potentiated by flagellin was characterized by the production of the Th2-specific cytokines IL-13 and IL-5 as well as the Th1-specific cytokine interferon- $\gamma$ (IFN- $\gamma$ ). Remarkably, the adjuvant effect was abrogated when CD4 T cells were depleted $24 \mathrm{~h}$ before i.n. immunization (data not shown). This is consistent with previous observations showing that flagellinmediated adjuvant effect by nasal route depends on CD4 T lymphocytes. ${ }^{18,32}$ Interestingly, flagellin in contrast to cholera toxin was not able to stimulate production of IL-17A and IL-22 by spleen or lymph node cells. These findings support the idea that flagellin activity on the mucosa enhances a mixed Th1-Th2 $\mathrm{T}$-cell response as well as IgA and IgG production in serum and secretions, a feature of mucosal adjuvants.

The activity of flagellin by i.n. route was found to depend on TLR5 as the adjuvant effect on B- and T-cell responses was abrogated in $T l r 5^{-1-}$ animals (Figure 2). In contrast to conventional animals, TLR5-deficient mice did not increase the systemic antibody response and the local antibody response (both $\operatorname{IgG1}$ and $\operatorname{IgA}$ ) specific for the antigen after nasal immunization with the flagellin-formulated vaccine (Figures $2 \mathbf{a}-\mathbf{c})$. Similar observations were done with respects to the
T-cell response as defined by the secretion of IFN- $\gamma$ and IL-13 by MdLN cells (Figures 2d and e). However, cholera toxin enhanced adaptive immune responses independently of TLR5 as both conventional and TLR5-deficient animals responded to nasal vaccination. We also assessed the contribution of NLRC4 in adjuvant activity of flagellin using recombinant flagellins harboring mutations in the C-terminal part as described previously. ${ }^{33}$ Administration of $\mathrm{FliC}_{492 \text { stop }}$ deficient in NLRC4 activation was as potent as the NLRC4 competent $\mathrm{FliC}_{\mathrm{V} 489 \mathrm{~A}}$ (harboring the same tag as $\mathrm{FliC}_{492 \text { stop }}$ ) or native flagellin in enhancing the adaptive response to OVA (Supplementary Figures 1D and E). Altogether, our results demonstrated that flagellin promotes TLR5-dependent adjuvant effects upon i.n. immunization independently of the NLRC4 signaling motif.

\section{Flagellin-mediated mucosal adaptive immunity requires radioresistant cells}

We hypothesized that the immune-adaptive responses induced by flagellin in the respiratory tract are mediated by the structural cells (mainly epithelial cells) as described for innate responses. ${ }^{28}$ To challenge this model, we generated radiation chimera by transplantation of bone marrow (BM). Irradiated wild type (WT, C57BL/6J) and TLR5-deficient mice were reconstituted with $\mathrm{BM}$ cells to obtain $\mathrm{Tlr} 5^{-1-} \rightarrow \mathrm{WT}$ and $\mathrm{WT} \rightarrow T \mathrm{Tl} 5^{-/-}$chimera and the control counterparts 

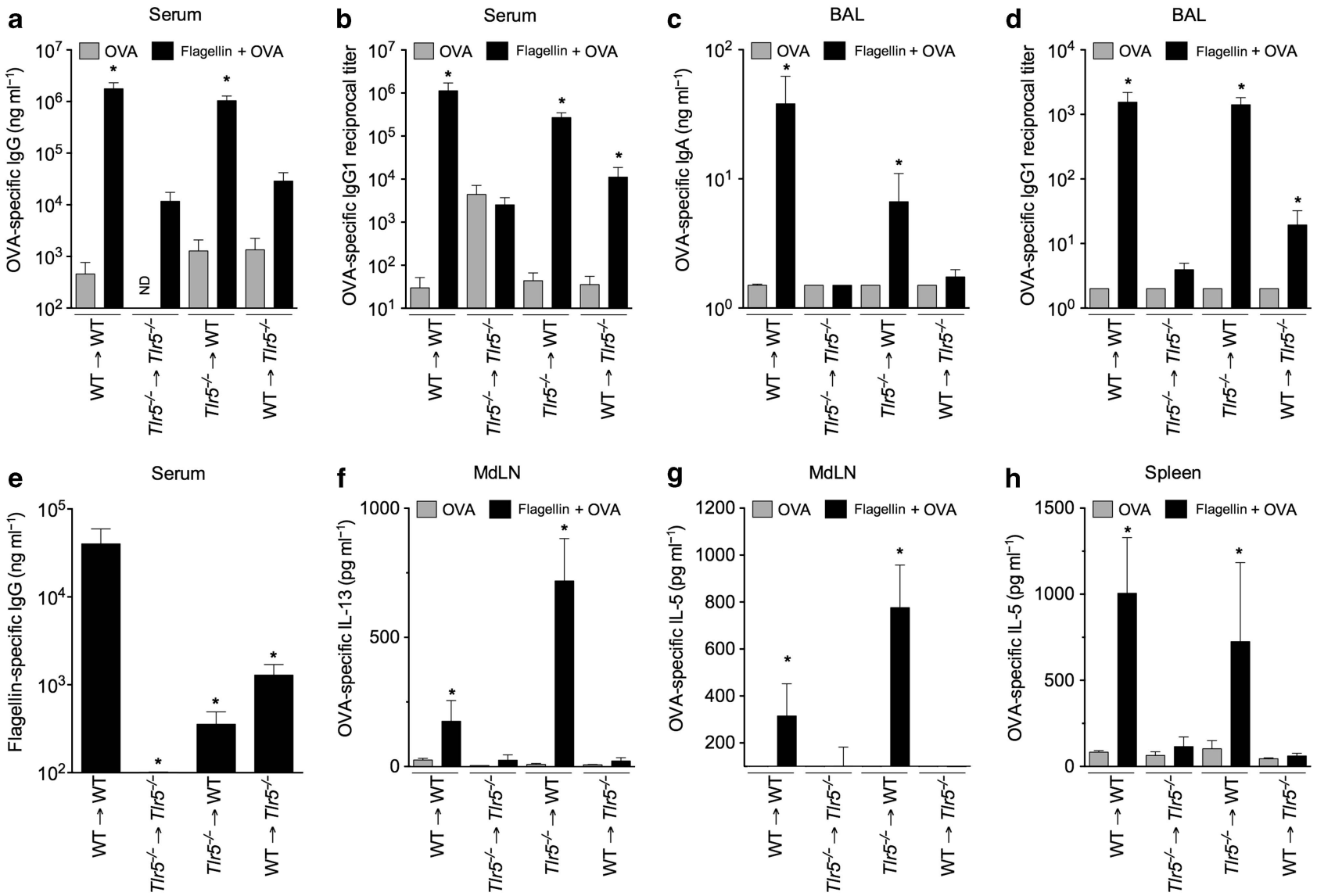

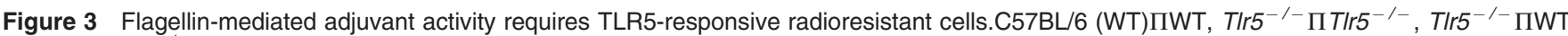
and WTחTIr5 $5^{-1-}$ bone marrow chimera mice $(n=7-14)$ were immunized intranasally at days 1 and 21 with ovalbumin (OVA) or flagellin + OVA in phosphate-buffered saline. Serum, bronchoalveolar lavage (BAL), spleen, and mediastinal lymph node (MdLN) were collected at day 35. (a-d) OVAspecific antibody response. Levels of OVA-specific IgG, IgG1 and IgA were determined by ELISA in serum (a, b) and BAL (c, d). (e) Flagellin-specific antibody response in serum. ( $\mathbf{f}-\mathbf{h})$ OVA-specific T-cell response. MdLN or spleen cells were stimulated $72 \mathrm{~h}$ with OVA and cytokine levels in supernatant were determined by ELISA. Statistical significance $\left({ }^{\star} P<0.05\right)$ was assessed by Mann-Whitney test with regard to OVA-vaccinated animals for each group (a-d and $\mathbf{f}-\mathbf{h}$ ) or WTחWT group (e). Results are expressed as mean \pm s.e.m. and are representative of 1-3 experiments. ND, not done.

$\operatorname{Tlr} 5^{-\prime-} \rightarrow \operatorname{Tlr} 5^{-\prime-}$ and $\mathrm{WT} \rightarrow \mathrm{WT}$. We determined that in Tlr $5^{-1-} \rightarrow$ WT chimera or Tlr $5^{-1-}$ mice, splenic DCs were responsive to LPS but not flagellin as defined by expression of CD86 and secretion of IL-12p40 in serum (Supplementary Figure 2). These results indicated that the hematopoietic compartment of $\mathrm{Tlr}^{-/-} \rightarrow$ WT mice including the DC compartment is deficient for flagellin/TLR5 signaling. Although the hematopoietic cells of $T l r 5^{-/-} \rightarrow$ WT were unable to respond to flagellin, the adjuvant activity of flagellin using nasal route of vaccination was not altered (Figure 3). For instance, circulating and secretory antibodies specific for the OVA antigen were significantly enhanced by flagellin i.n. vaccination in $T l r 5^{-1-} \rightarrow \mathrm{WT}$ animals compared with the group immunized with standalone antigen formulation (Figures 3a-d). Analysis of cytokine production by OVAstimulated MdLN and spleen revealed that $T l r 5^{-\prime-} \rightarrow$ WT animals were also potently activated by the nasal vaccines containing flagellin (Figures 3f-h). As Tlr $5^{-/-} \rightarrow \mathrm{WT}$ were effectively reconstituted by donor cells ( $>96 \%$ blood cells, $>90 \%$ lung hematopoietic cells, and $>95 \%$ alveolar macrophages), we concluded that the adaptive immune response triggered by the mucosal adjuvant flagellin is mainly regulated by TLR5 signaling in the radioresistant compartment. Conversely, the WT $\rightarrow T l r 5^{-1-}$ mice elicited lower T-cell-mediated and antibody responses compared with vaccination with flagellin and OVA. We found that antibody response and cytokine responses were mostly abrogated or similar to the response of $T l r 5^{-/-} \rightarrow T l r 5^{-/-}$mice. Our data showed also that the antibody response specific for flagellin was strongly impaired in the various chimera compared with the positive control WT $\rightarrow \mathrm{WT}$ (Figure 3e). As one might expect, the antibody response in $T l r 5^{-1-} \rightarrow$ WT chimera was dominated by the IgG1 isotype as described for conventional animals and Th2-type cytokines, and was associated to production of secretory antibodies like IgA. Altogether, our results showed that radioresistant cells are crucial to establish the adaptive immune response to flagellin in lung mucosa.

We previously demonstrated that either flagellin-specific "hyperimmunization" of animals or passive transfer of antiflagellin serum from "hyperimmune" animals is able to neutralize the systemic activity of flagellin on innate signaling. ${ }^{18}$ 
Using this approach, we addressed whether flagellin needs to access the systemic compartment to promote adjuvant activity by i.n. route (Supplementary Figure 3). We first found that flagellin-specific circulating antibodies impaired the activation of splenic DCs after intravenous (IV) administration of flagellin in contrast to control antibodies (Supplementary Figure 3A). Similarly, the antibodies against flagellin totally blocked the potency to induce the systemic proinflammatory response as assessed with the CCL20 response (Supplementary Figure 3B). Using the same conditions of neutralization, the capacity of flagellin to increase the OVA-specific antibody response was shown to be impaired after IV vaccination with flagellin \pm OVA (Supplementary Figure 3C). However, the neutralizing antibodies did not affect the systemic response to the TLR3 agonist poly I:C, demonstrating specificity of these findings. Using i.n. vaccination route, we found that flagellin-specific circulating neutralizing antibodies were not able to alter the mucosal adjuvant activity of flagellin (Supplementary Figure 3D). These data suggest that the mucosal adjuvant functions of flagellin do not require the flagellin-mediated innate signaling in the resident hematopoietic cells of lung parenchyma, blood, or peripheral tissues. Altogether, our experiments support the idea that structural cells of airways are the key targets of TLR5 signaling driving the mucosal adjuvant activity.

\section{Flagellin is compartmentalized to the conducting airways} and is rapidly degraded

To further define the mechanisms of action of adjuvants, the distribution of flagellin was investigated in the respiratory tract. We took advantage of the fact that systemic delivery of flagellin triggers an immediate IL-22 production in a process fully dependent on TLR5 signaling in DCs. ${ }^{23,24}$ As expected, flagellin activated within $2 \mathrm{~h}$ a swift and transient production of IL-22 in the respiratory tract, in blood and in spleen when delivered by the IV route (Figures $4 \mathbf{a}$ and $\mathbf{b}$ ). In contrast, i.n. administration of a potent dose of flagellin adjuvant was not associated with a significant modulation of IL-22 secretion or transcription, both in airway parenchyma, blood, and spleen. These results were not due to delay of flagellin delivery from airways into the parenchyma or systemic compartment as the unresponsiveness was similar at $6 \mathrm{~h}$ post instillation. Although i.n. delivery of flagellin did not trigger Il22 expression in lungs, it stimulated a strong proinflammatory response in lung tissue (Figure 5). These results suggested that flagellin is not translocated into the parenchymal compartment of lungs, blood, or peripheral tissues.

We next addressed how flagellin was processed in the airways. Lavages of the conducting airways (BAL) and lung tissue extracts were analyzed at various times post administration for degradation and biological activity using flagellinspecific immunoblot and ELISA (Figures $\mathbf{4 c - g}$ ). We first found that flagellin was recovered in the BAL immediately after i.n. administration (about $10-20 \%$ of the initial input). In contrast, only $1 \%$ flagellin was recovered from the lung tissue and this amount decreased during time, indicating that flagellin was mainly associated with the conducting airways (Figure 4d).
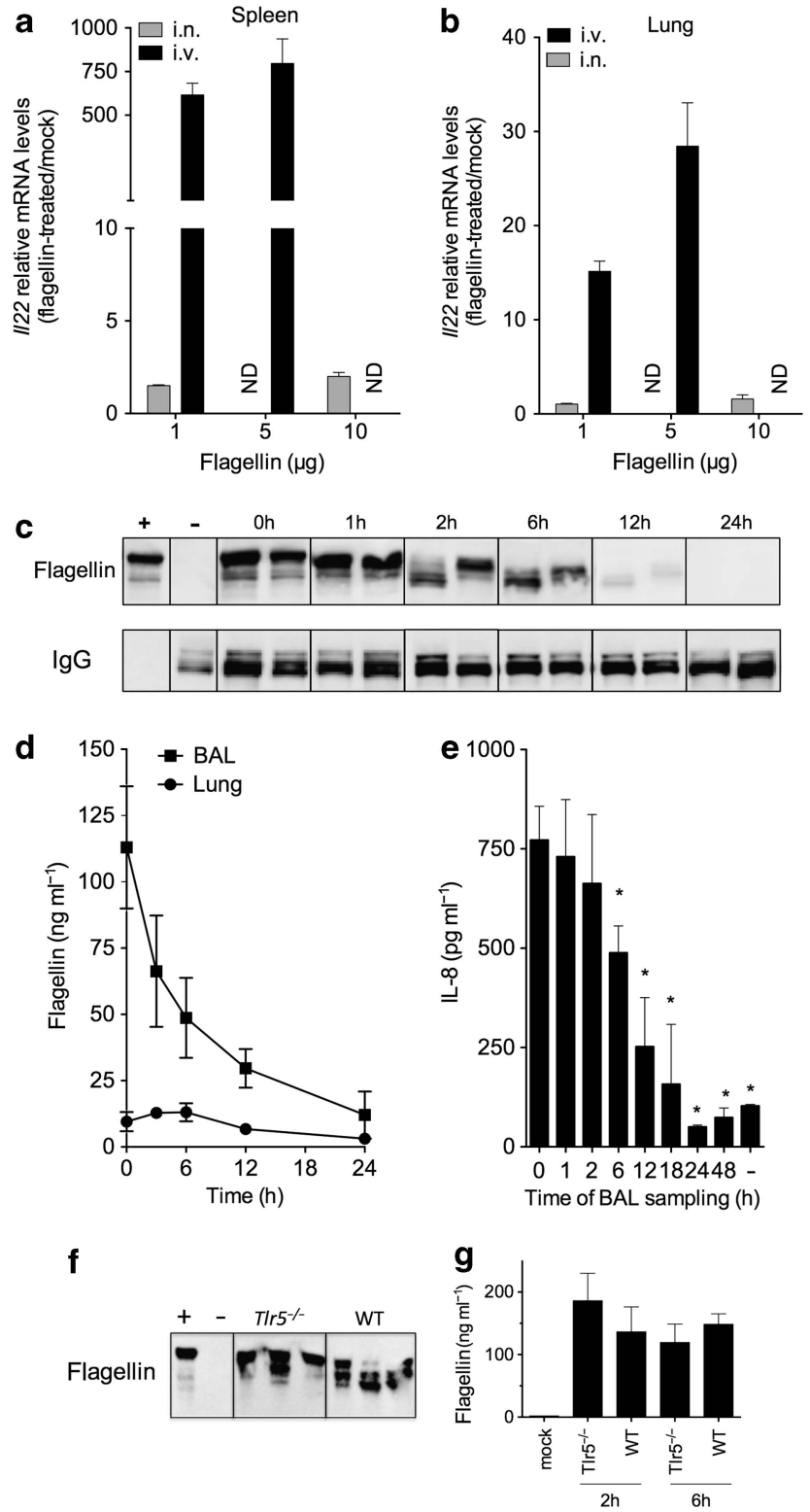

Figure 4 Flagellin is rapidly and totally degraded in airways conducts. C57BL/6 mice $(n=3-6)$ were treated intravenously or intranasally (i.n.) with flagellin. Spleen (a) and lung (b) were sampled at $2 \mathrm{~h}$ and II22 mRNA levels were determined by quantitative PCR. Levels of $m R N A$ in mock animal were arbitrarily set to 1 and used to calculate relative gene expression. (c-g) Mice $(n=3-4)$ were stimulated i.n. with $1 \mu \mathrm{g}(\mathbf{d}, \mathbf{e}, \mathbf{g})$ or $10 \mu \mathrm{g}(\mathbf{c}, \mathbf{f})$ flagellin and lungs and bronchoalveolar lavage (BAL) of mock or flagellin-treated mice were sampled at indicated time. " - " and " + " indicate BAL from mock animals and BAL supplemented ex vivo with flagellin, respectively. In panels c-e experiments were conducted on NMRI mice, whereas $T / r 5^{-1-}$ and C57BL/6 (WT) animals were used for panels (f, g). (c) Kinetic of flagellin degradation. Flagellin (upper panel) or endogenous IgG (lower panel) were analyzed by SDS-polyacrylamide gel electrophoresis and immunoblot. For each time, the immunoblot for two animals were shown. (d) Concentration of flagellin was estimated by ELISA. (e) Time course analysis of flagellin activity in sampled BAL. Epithelial cells Caco-2 were stimulated $24 \mathrm{~h}$ with BAL isolated from flagellin-treated mice sampling at indicated time or medium as control $(-)$. Levels of IL-8 in supernatants were determined by ELISA.

Results are expressed as mean \pm s.d. Statistical significance $\left({ }^{*} P<0.05\right)$ was assessed by Mann-Whitney test compared with phosphate-buffered saline $(\mathbf{a}, \mathbf{b})$ or $\mathrm{t}_{0}(\mathbf{e})$ group. (f) Flagellin degradation in BAL $2 \mathrm{~h}$ after nasal administration was analyzed by flagellin-specific immunoblot. For each group, the immunoblot for 3 animals were shown. (g) Concentration of flagellin was estimated by ELISA at 2 and $6 \mathrm{~h}$. ND, not done. 


\begin{tabular}{|c|c|l|}
\hline \multicolumn{2}{|c|}{ Group } & \multicolumn{1}{c|}{ Genes } \\
\hline I & Immune mediators & $\begin{array}{l}\text { Cc/3, Ccl20, Ccrl2, Cxcl1, Cxcl2, Cxcl10, II1a, Tnf } \\
\text { Cd83, Csf2 }\end{array}$ \\
\hline II & $\begin{array}{c}\text { Transcriptional } \\
\text { regulation of innate } \\
\text { immunity }\end{array}$ & Fos, Fosb, Jun, Junb, Nfkbiz \\
Atf3, Nfkbia, Tnfaip3, Zfp36,
\end{tabular}
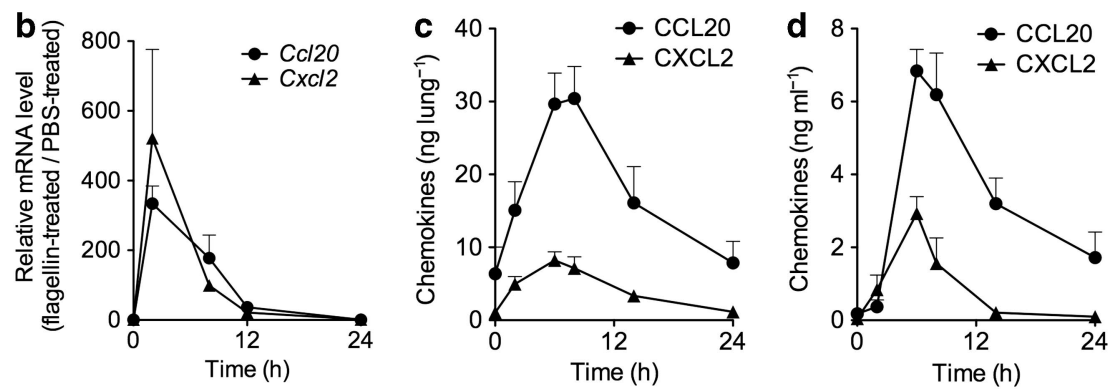

Figure 5 TLR5 activation promotes an early and transient lung proinflammatory signature. (a) Transcriptomic analysis of lung response to flagellin. $\mathrm{C} 3 \mathrm{H} / \mathrm{HeJ}$ mice ( $n=9$ /group) were treated intranasally (i.n.) with phosphate-buffered saline (PBS) or flagellin. Total lung RNA was extracted $1 \mathrm{~h}$ later and processed for hybridization on microarrays. Genes differentially expressed (fold increase $>1.5$ and $P<0.05$ ) after $1 \mathrm{~h}$ of flagellin treatment were arbitrary classified in three groups depending on their biological function. (b-d) Time-course analysis of pulmonary response to flagellin. C57BL/6 mice $(n=4-6)$ were treated i.n. with PBS or flagellin. At indicated times, lung (b, c) or bronchoalveolar lavages (BAL) (d) were sampled. Levels of Ccl20 and Cxcl2 mRNA were analyzed by quantitative PCR (b) and levels of chemokines CCL20 and CXCL2 (c, d) were determined by ELISA. Levels of mRNA in PBS-treated animals were arbitrarily set to 1 and used to calculate relative gene expression. Results are expressed as mean $\pm s$.d. for mRNA and mean $\pm s$.e.m. for proteins and are representative of 2-3 experiments.

The low amounts of lung-associated flagellin represented either transepithelial-translocated flagellin or flagellin attached to the epithelium. Flagellin was degraded within hours in BAL, whereas the total BAL IgG used as a surrogate indicator of quality of samples were stable (Figures $4 \mathrm{c}$ and d). First signs of proteolysis were observed as soon as $1 \mathrm{~h}$ post administration and at $12 \mathrm{~h}$ most protein was fully degraded. In addition, the degradation in BAL was found to correlate with the concomitant decrease of capacity of flagellin to induce innate signaling, as defined on the Caco-2 cells that are potent responders to TLR5-dependent signaling (Figure 4e). These data are consistent with the susceptibility of native flagellin to protease activity. ${ }^{34,35}$ Finally, we determined that the susceptibility of flagellin to degradation was similar in Tlr5 $5^{-1-}$ animals and the TLR5-sufficient controls (Figures $4 \mathbf{f}-\mathbf{g}$ ), suggesting that absence of TLR5 does not impact on flagellin degradation in airways. In conclusion, our experiments showed that after nasal administration, flagellin is mainly restrained to the conducting airways and is rapidly degraded to inactive peptides by airways proteases.

\section{Flagellin activates a gene expression program specific for} regulated mucosal immune responses

TLR signaling is characterized by a swift and strong transcriptional activation. To further define the compartments where activation takes place, microarray experiments were performed on whole lungs $1 \mathrm{~h}$ after flagellin administration, a short period that minimized bias consequent to tissue remodeling and immune cell infiltration (Figure 5 and Supplementary Figure 4). ${ }^{28}$ Although, our flagellin preparations were LPS-depleted ( $<20$ pg LPS per $\mu$ g flagellin), these experiments were conducted on the TLR4 hyporesponsive $\mathrm{C} 3 \mathrm{H} / \mathrm{HeJ}$ animals to rule any effect of low levels of LPS contamination traces on gene expression. The analysis identified 34 genes that were differentially upregulated by flagellin and were part of signaling pathways including TLR, inflammation mediated by chemokine and cytokine, TGF- $\beta$, and apoptosis. We classified the flagellin-activated genes into three groups according to their biological function. The first group of genes encodes cytokines like IL- $1 \alpha$ and tumor necrosis factor- $\alpha$, chemokines like CCL3, CCL20, CXCL-1, 2, 10 as well as $\mathrm{CD} 83$ and granulocyte macrophage colony-stimulating factor associated with DC activation/differentiation. Interestingly, the chemokine CCL20 is known to participate in the recruitment of DC precursors. ${ }^{36}$ The second group includes genes encoding transcriptional activators of innate signaling such as JUN, FOS, and I- $\kappa \mathrm{B} \zeta$ and negative regulators of TLR activation like TNFAIP3 (A20), I- $\kappa \mathrm{B} \alpha$, and tristetraprolin (ZFP36). This feature suggests that the lung response to TLR5 agonist is a highly regulated process. The third group of genes encodes factors involved in differentiation, repair, and resistance of cells and tissues to stress and apoptosis. This set of genes may limit lung damage caused by cellular infiltration and promote a rapid feedback to homeostasis. Microarray data were validated by quantitative PCR gene expression analysis and the correlation was highly significant (Supplementary Figures $4 \mathrm{~A}-\mathrm{C}$ ). This signature was not 
specific of $\mathrm{C} 3 \mathrm{H} / \mathrm{HeJ}$ mice as similar patterns of activation were defined in $\mathrm{C} 57 \mathrm{BL} / 6, \mathrm{BALB} / \mathrm{c}$, and the outbred NMRI mice (Supplementary Figure 4E).

Next, the kinetics of the lung response to flagellin was investigated. We determined the gene expression and protein levels in lung (Figures 5a-b) and BAL (Figure 5c) of chemokines CXCL2 and CCL20. A swift and transient transcription and secretion was observed with a peak at 2 and $6 \mathrm{~h}$, respectively. Interestingly, the steady-state expression was mostly reinstated at $24 \mathrm{~h}$. Such patterns of expression were found for most genes of the signature (Supplementary Figure 4D). Remarkably, this transient response was observed in prime (day 1) and prime-boost experiments (day 1 and day 21), indicating that similar innate mechanisms are triggered by flagellin during nasal vaccination independently of any memory adaptive response (Supplementary Figure 4F). Therefore, the lung-specific TLR5 signature showed that flagellin stimulates a highly regulated response signal.

Finally, the contribution of radioresistant and hematopoietic compartments to TLR5 signature was addressed using BM chimera. To this aim animals were left untreated (mock) or treated i.n. with flagellin and the level of transcriptional activation was assessed by quantitative reverse-transcriptasePCR. We observed that flagellin-dependent transcription of several genes of the TLR5-specific lung signature was fully impaired in Tlr $5^{-1-} \rightarrow T l r 5^{-1-}$ mice compared with that in $\mathrm{WT} \rightarrow \mathrm{WT}$ chimera (Figure 6). Moreover, the transcriptional activation stimulated by flagellin was consistently higher in Tlr $5^{-1-} \rightarrow$ WT than in Tlr5 $5^{-1-} \rightarrow$ WT. In conclusion, our results show that radioresistant cells are key players for stimulation of TLR5-dependent transcription. These data also highlighted a strong correlation between the localization of flagellin within the conducting airways, the early mucosal transcriptional response stimulated by flagellin and the mucosal adjuvant potency of flagellin-containing vaccines.

\section{Respiratory epithelial cells are the main responder cells to TLR5 stimulation}

The lung bronchoalveolar compartment is mainly constituted by radioresistant structural cells (epithelial cells) and resident macrophages. Our experiments showed that the pulmonary and alveolar macrophages are poorly responsive to flagellin in contrast to CpG (TLR9) or LPS (TLR4) (Supplementary Figure 5 and data not shown). For instance, macrophages isolated from alveoli did not produce any IL-12p40 after flagellin stimulation, whereas a significant but low level of IL12p40 production was stimulated in pulmonary macrophages. This is also consistent with data obtained with peritoneal and BM-derived macrophages that are poorly activated by flagellin in contrast to LPS or CpG, although a significant but moderate activation of nuclear factor $(\mathrm{NF})-\mathrm{\kappa B}$ signaling (Supplementary Figure 5). As flagellin induced a fast signaling, was not translocated to parenchyma, did not activate in a significant manner the alveolar hematopoietic cells, and was functional on the radioresi- stant compartment, we investigate the contribution of structural cells to the signaling. To examine this hypothesis, microdissection of lung from phosphate-buffered saline (PBS)or flagellin-treated mice was performed to enrich bronchial epithelium or whole alveolar compartment and to achieve quantitative gene expression analysis with regard to the TLR5 signature (Figures $7 \mathbf{a}$ and $\mathbf{b}$ ). Interestingly, the magnitude and the pattern of the bronchial epithelium transcriptional response to flagellin were similar to the response of the entire lung. A similar transcriptional pattern was found for the whole alveolar compartment but to a lower extent. In addition, we found that alveolar and tracheal cells isolated from mice are stimulated $>50$ fold with regard to Ccl20 expression in response to flagellin (data not shown). This is in agreement with recent observations indicating that mouse respiratory epithelial cells are the main cells expressing Tlr $5 \mathrm{mRNA}$ and responding to flagellin upon nasal administration. ${ }^{19}$ Furthermore, the gut response was investigated upon luminal administration of flagellin into ligated ileal loops (Supplementary Figure 6). In these experiments, Ccl20 expression was also exclusively restricted to the intestinal epithelium. To get further insights, we studied the response of human epithelial cell lines to flagellin stimulation. Expression of TLR5 signaturespecific genes including CCL20, CXCL1, CXCL2, IL8, NFKBIA, NFKBIZ, TNF and TNFAIP3 was strongly upregulated in the respiratory epithelial cells including BEAS-2B or 16HBE (bronchial) and A549 (alveolar) (Figures $7 \mathrm{c}$ and d). We found that after $24-\mathrm{h}$ stimulation by flagellin, BEAS2B and $16 \mathrm{HBE}$ secreted significant amounts of CCL20 (flagellin: $563 \pm 63$ and $642 \pm 53 \mathrm{pg} \mathrm{ml}^{-1}$ versus controls: $6 \mathrm{pg} \mathrm{ml}^{-1}$ ) and IL-8 (flagellin: $1291 \pm 219$ and 4,185 $\pm 339 \mathrm{pg} \mathrm{ml}^{-1}$ versus controls: 6 and $380 \pm 52 \mathrm{pg} \mathrm{ml}^{-1}$ ) and whereas tumor necrosis factor was not found in BEAS-2B and barely detectable in $16 \mathrm{HBE}$. These results indicated that respiratory epithelial cells recapitulate the whole lung response. In conclusion, this study suggests that the epithelial compartment drives the activity of the mucosal adjuvant flagellin.

\section{DISCUSSION}

This study demonstrates that TLR5 signaling in airway structural cells drives the flagellin-specific mucosal adjuvant activity on adaptive immunity. We showed that the i.n. administration of flagellin activates antibody- and T-cellmediated immune responses in the systemic and respiratory compartments. The adjuvant activity was dependent on TLR5 but not on NLRC4 and correlated with TLR5 signaling in radioresistant cells. Furthermore, flagellin was rapidly degraded in the respiratory tract, indicating that TLR5 signaling occurs swiftly in the airway epithelial compartment. Bronchial and alveolar epithelial cells mostly recapitulated the flagellinmediated transcriptional activation of mouse lung tissue. In conclusion, these data suggest that the airway epithelial cells regulate the adjuvant activity of flagellin.

Various studies highlighted the contribution of epithelium to immunity in response to microbes or microbial motifs. ${ }^{6,27}$ Pioneer in vitro works showed that intestinal epithelial cells in 

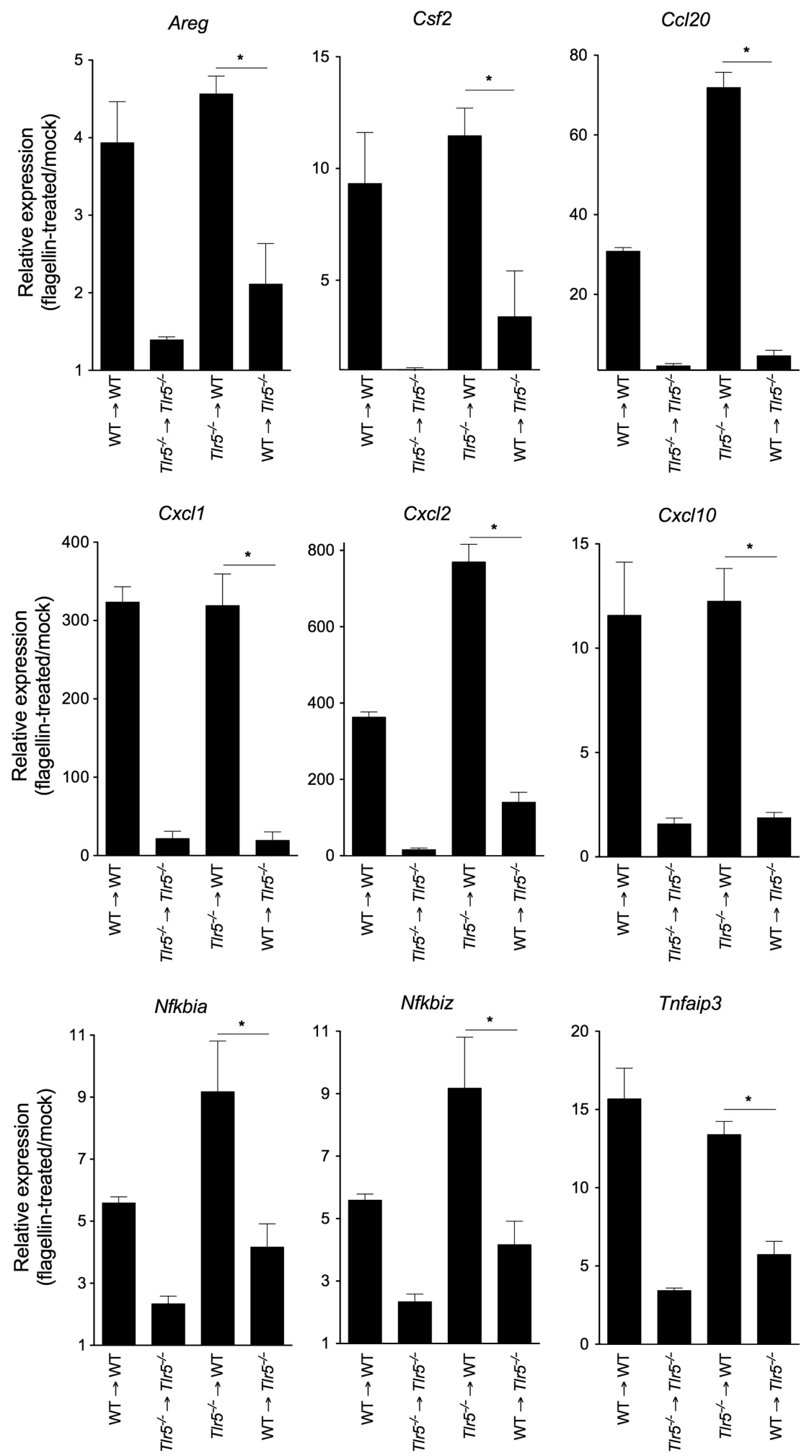

Figure 6 Lung-specific TLR5 signature depends on signaling in radioresistant cells. C57BL/6 (WT) $\Pi$ WT, TIr $5^{-1-} \rightarrow$ TIr $5^{-1-}$, TIr $5^{-1-} \rightarrow$ WT and $\mathrm{WT} \rightarrow \mathrm{TIr} 5^{-/-}$bone marrow chimera mice $(n=3-4)$ were treated intranasally with flagellin or untreated. Lungs were sampled at $2 \mathrm{~h}$ and mRNA levels of various proinflammatory genes were analyzed by quantitative PCR. Levels of mRNA in mock animals were arbitrarily set to 1 and used to calculate relative gene expression of flagellin-treated animals. Results are expressed as mean \pm s.e.m. Statistical significance $\left({ }^{\star} P<0.05\right)$ was assessed by Mann-Whitney test between TIr5 ${ }^{-1-} \rightarrow$ WT and WT $\rightarrow$ TIr $5^{-1-}$ groups.

response to bacteria produce proinflammatory mediators like the CXCL8 chemokine, and allow the recruitment of neutrophils into the gut lumen. ${ }^{37,38}$ Similar observations were carried out with bronchial and alveolar epithelial cells. ${ }^{39}$ In vivo, contribution of gut and airway epithelial cells was supported by experiments performed with chimera or tissue-specific 
deficient mice. ${ }^{28,40-42}$ Similarly, TLR5-mediated mucosal innate immunity was found to depend on signaling within the epithelium compartment of mucosa. ${ }^{28,43}$ Stromal and
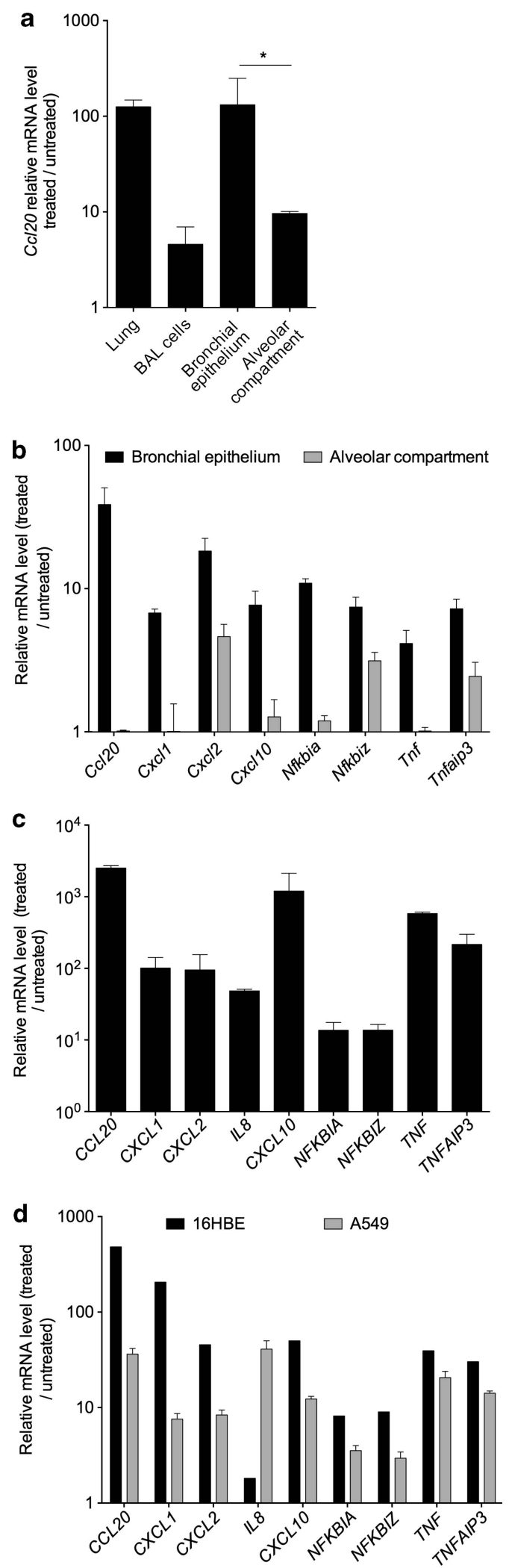

hematopoietic cells can crosstalk to provide effective adaptive responses upon TLR stimulation. ${ }^{44,45}$ The epithelial or stromal TLR signaling was associated with adaptive immunity due to the production of mediators modulating immune cells like DCs or lymphocytes. ${ }^{6,27}$ TLR-stimulated epithelium can regulate DC activation via thymic stromal lymphopoietin, ${ }^{46}$ or can condition antibody responses by production of B-cell-specific cytokines like BAFF and APRIL. ${ }^{47}$ In response to bacterial or TLR agonist, epithelial CCL20 stimulation attracts immature DC precursors or lymphocytes. ${ }^{25,48}$ Le Borgne et al. ${ }^{36}$ showed that CCR6-dependent APC recruitment is required to trigger adaptive immunity using microbial compounds as adjuvant for cutaneous or buccal route. The present study demonstrated that airway epithelial cells produce CCL20 upon nasal administration of flagellin. In the same way, the production of granulocyte macrophage colony-stimulating factor is a major activator of lung DC activity on antigen-specific immune responses. ${ }^{49}$ Recent studies highlighted the contribution of epithelial thymic stromal lymphopoietin, IL-25, and IL-33 in the regulation of allergy in response to stimulation with antigen and TLR4 or TLR5 agonists. ${ }^{19,41}$ Recruitment and/or activation of lung APC by epithelial mediators may therefore represent the mechanism promoting mucosal adjuvant activity of flagellin. Next, investigations have to define the nature of APCs, i.e., monocyte or DC, that are involved in the sampling of antigen through epithelial barrier and in the antigen presentation in draining lymph nodes.

It is assumed that adjuvant activity of TLR agonists like the monophosphoryl lipid A used in some commercial vaccines depends on direct TLR-mediated signaling in APC. Reis e Sousa et al. demonstrated that the systemic administration of TLR agonists activates the adaptive immune response in a process that strictly depends on TLR-mediated activation of APC in lymphoid organs. ${ }^{8,50}$ Flagellin, like other TLR agonists, activates APC in a TLR5-dependent manner when administered by systemic route. ${ }^{15,24,40}$ Here we demonstrated that adjuvant activity of flagellin when delivered by nasal route is not affected even though TLR5 signaling is deficient in hematopoietic cells or flagellin activity is neutralized in the systemic compartment. This work therefore supports the hypothesis that adaptive immune responses induced by TLR agonists can result

Figure 7 Airway epithelial cells replicate the lung signature to flagellin (a, b) Transcripts analysis of microdissected lung compartments. C57BL/6 mice $(n=2-3)$ were treated i.n. with phosphate-buffered saline or flagellin $2 \mathrm{~h}$. Bronchoalveolar lavage (BAL) and lungs were sampled. Lungs were prepared for laser microdissection of bronchial epithelium and alveolar compartment. Level of Ccl20 mRNA (a) and others proinflammatory genes transcripts (b) in bronchial epithelium and whole alveolar compartments, BAL cells or total lung were determined by quantitative PCR (qPCR). (c, d) Activation of human epithelial cells by flagellin treatment. Normal bronchial epithelial cell line BEAS-2B were stimulated $1 \mathrm{~h}$ with flagellin (c). Bronchial and alveolar epithelial cell line, $16 \mathrm{HBE}$ and A549, respectively, were stimulated $2 \mathrm{~h}$ with flagellin (d). Total RNA was extracted and proinflammatory gene expression analyzed by qPCR. Levels of mRNA in mock animals or cells were arbitrarily set to 1 and used to calculate relative gene expression of flagellin-treated animals or cells. Results are expressed as mean \pm s.d. Statistical significance $\left({ }^{\star} P<0.05\right)$ was assessed by Mann-Whitney test. 
from indirect APC activation by TLR-induced signals in structural cells. This feature may be specific for non-lymphoid tissues like lungs that harbor (and recruit) APC specifically conditioned by the environment, thereby distinguishing these APCs from lymphoid organ APCs. Moreover, non-lymphoid tissues are prone to strong proinflammatory signaling due to structural cells that impact on APCs and adaptive immune responses. Indeed, CCL20 and granulocyte macrophage colony-stimulating factor represent potent conditioning epithelial factors of APCs as they promote APC recruitment or differentiation in non-lymphoid tissues. ${ }^{36,49}$ Recently, Hammad et al. ${ }^{41}$ proposed that epithelial cells are sufficient to drive T-cell responses in a TLR4-dependent stimulation of the respiratory tract. Understanding how TLR4 and TLR5 condition the epithelium to manipulate signals needed for recruitment and activation of APCs will be a major topic of future research.

This study indicates that the TLR5-stimulating motif of flagellin but not the NLRC4 motif is required for mucosal adjuvant activity. In contrast, Vijay-Kumar et al. ${ }^{17}$ showed that both TLR5 and NLRC4 could contribute to the adaptive immune responses induced by flagellin by the systemic route. These results suggest that the immunization route conditions the mode of action of adjuvants.

Most adjuvants enhance immunogenicity of antigens by eliciting a proinflammatory environment that recruits phagocytic cells. Major issues are therefore the induction of sustained inflammation. ${ }^{1}$ The i.n. administration of flagellin induces a transient proinflammatory response into the lung. $\mathrm{NF}-\kappa \mathrm{B}$ is an important regulator of TLR-mediated responses that has to be controlled in a timely manner as excessive or sustained signaling can cause tissue damage. Our analysis showed a rapid upregulation of the expression of genes encoding the TLR- and NF- $\kappa \mathrm{B}$-negative regulators $\mathrm{A} 20, \mathrm{ZFP} 36, \mathrm{I} \kappa \mathrm{B} \alpha$, and ATF $3 .^{51}$ These regulators act along the TLR signaling pathway, thereby blocking untimely or prolonged proinflammatory responses. The deubiquitinase A20 inactivates the TLR signaling cascade. ${ }^{52,53}$ Recent data indicated that epithelial expression of A20 is crucial to contain mucosal inflammation, especially in the gut by protecting enterocytes from apoptosis and resolving inflammation. ${ }^{54}$ Similarly, de novo synthesis of $\mathrm{I}-\kappa \mathrm{B} \alpha$ prevents nuclear localization of NF- $\kappa \mathrm{B} .{ }^{51}$ The transcriptional repressor ATF3 inhibits the TLR-dependent transcription of proinflammatory genes like Il6, Cxcll, and Cxcl2, particularly in the respiratory tract. ${ }^{55}$ The posttranscriptional regulator tristetraprolin encoded by Zfp36 is known to destabilize the mRNA of Tnf or Csf2. ${ }^{51,56}$ Interestingly, after primary flagellin administration, the lung tissue was not able to respond for $24 \mathrm{~h}$ to a secondary stimulation (data not shown). The negative regulators identified in this study could thus participate in the process of tolerance. Finally, the rapid degradation of flagellin is also an important feature that promotes attenuation of TLR signaling.

Besides regulation of TLR-mediated inflammatory signaling, the capacity of an adjuvant to induce rapid restoration of steady-state homeostasis is an important feature. Interestingly, the nasal instillation of flagellin activated expression of genes required for tissue and cellular repair, and limiting inflammation, including EGF-like growth factor amphiregulin, the DNA repair protein GADD $45 \beta$ and the TGF $\beta$ family factor GDF15. Recent studies showed that flagellin stimulates survival and proliferation of epithelial intestinal cells after abrasion of cell monolayers. ${ }^{57}$ Beyond the role in adjuvant activity, activation of local repair mechanisms may be crucial for the therapeutic potential of flagellin in various models, including respiratory infections or systemic pathologies. ${ }^{58,59}$

In conclusion, our data provided new understanding of the mode of action of vaccine adjuvants. We defined that mucosal adjuvant activity can be driven by signaling in airway epithelial cells. This concept of an epithelial adjuvant may be generalized to other adjuvants, and to the screening of new adjuvant molecules. To this aim, it will be essential to dissect the mechanisms that promote TLR-mediated adjuvant activity at mucosal surfaces. Future experiments will need to identify signals from epithelial cells involved in the recruitment and the activation of APCs as well as the nature of APCs driving the adjuvant effect. Finally, it is crucial to define whether these observations are specific for flagellin and the respiratory tract or can be extended to unrelated adjuvants or mucosa as suggested by our experiments in the gut.

\section{METHODS}

Mice. Six-to-ten-week old C57BL/6J, C3H/HeJ, or NMRI mice were purchased from Charles River Laboratories (Lyon, France) or Janvier (Le Genest-Saint-Isle, France); Tlr $5^{-/-}$backcrossed on C57BL/6J genetic background and congenic C57BL/6J-CD45.1 were maintained in a specific pathogen-free facility (\#A59-350009, Institut Pasteur de Lille; Transgenose Institute CNRS, Orleans, France) and experiments complied with national regulations and ethical guidelines (protocol CEEA 052011).

Immunization. Mice were anesthetized by intraperitoneal injection of ketamine-xylazine or gaseous isoflurane and were administered with $20 \mu \mathrm{l}$ of PBS \pm antigens and adjuvants by i.n. route. Mice were vaccinated i.n. on days 1 and 21 with endograde ovalbumin (OVA; 10 or $20 \mu \mathrm{g}$, Hyglos) \pm LPS-depleted Flagellin FliC from Salmonella enterica Serovar Typhimurium produced as described ${ }^{18,25}$ $(1 \mu \mathrm{g}) \pm$ cholera toxin $(1 \mu \mathrm{g}$, Sigma, St Louis, MO). Bronchoalveolar lavages (BALs), mediastinal lymph nodes (MdLNs), lung, spleen, and serum were sampled on day 35 . BALs were collected after the intratracheal injection of $1 \mathrm{ml}$ PBS with Protease Inhibitor Cocktail (Roche, Basel, Switzerland). Lung protein extracts were prepared by homogenizing tissue in Tissue Protein Extraction Reagent (Pierce, Rockford, IL) supplemented with protease inhibitors. When indicated, vaccines were administered by IV route with $200 \mu \mathrm{l}$ of PBS \pm antigens and adjuvants.

Flagellin distribution. BALs were sampled and BAL proteins $(25 \mu \mathrm{g})$ were separated on SDS-polyacrylamide gel electrophoresis and blotted on a nitrocellulose membrane (Amersham, Piscataway, NJ). Flagellin was detected using rabbit anti-FliC polyclonal $\mathrm{IgG}^{18}{ }^{18}$ horseradish peroxidise (HRP)-conjugated Goat anti-Rabbit IgG, and chimioluminescent HRP reagents. Concentrations of flagellin in BAL and lung extracts were defined by ELISA. Plates were coated with mouse antiFliC IgG $\left(10 \mu \mathrm{g} \mathrm{ml}^{-1}\right.$ in PBS). Detection was performed using the antiFliC antibodies conjugated to biotin, HRP-conjugated Streptavidin (GE Healthcare, Piscataway, NJ) and the chromogenic reagent TMB. Standard curves were used to estimate the concentration of flagellin. 
BM chimera. Recipient mice were irradiated (10-15Gy) and reconstituted 2-24-h later with BM cells $\left(5-10 \times 10^{6}\right.$ cells IV). The mice were analyzed 3 months after BM transplantation and the degree of chimerism was assessed by measuring CD 45.1 and CD 45.2 expression by leukocytes using flow cytometer as described. ${ }^{23}$ The protocol yielded $>96 \%$ of reconstitution in the blood; $>90 \%$ for Tlr $5^{-\prime-}$ WT and $>95 \%$ for WT Tlr $5^{-\prime-}$ in lung hematopoietic cells; $>95 \%$ for Tlr $5^{-1-}$ WT and $>83 \%$ for WT Tlr $5^{-1-}$ in F $4 / 80^{+}$ alveolar macrophages.

Antigen-specific immune responses. Levels of OVA-specific antibodies in serum and BAL samples were assessed by ELISA as described. ${ }^{18}$ A reference serum has been included into individual plates to ensure reproducibility of titer calculations. Titers are given as geometrical means of titers from individual mice. When indicated, the concentration of immunoglobulin was defined using a standard curve with purified IgG or IgA (Sigma). OVA-specific T-cell responses were analyzed in mice 10-14 days after the last immunization. Spleen and MdLN cells $\left(2-5 \times 10^{6}\right)$ were incubated for $72 \mathrm{~h}$ with RPMI- 5 OVA $\left(1 \mathrm{mg} \mathrm{ml}^{-1}\right)$ to measure secretion of IL-5, IL-13, IL-17A, IL-22, and IFN- $\gamma$.

Cytokine production. Cytokine and chemokine levels were measured in serum, BAL, lung extract, or cell culture supernatant using commercial ELISA kits: R\&D Systems (Abingdon, UK; IL-8, CCL20, and CXCL2); eBioscience (San Diego, CA; IL-13, IL-17A, IL-22, and IFN- $\gamma$ ); or Becton Dickinson (Lincoln Park, NJ; IL-5 and IL-12p40).

Gene expression. Total RNA from mouse microdissected lung compartments, whole lung, BAL, or cultured cells was extracted with Nucleospin RNA kits (Macherey-Nagel, Duren, Germany). RNA was reverse-transcribed with the High-Capacity cDNA Archive Kit (Applied Biosystems, Foster City, CA). A preamplification of cDNA from microdissected compartments was performed before real-time PCR using the PreAmp kit (Applied Biosystems). cDNA was amplified using SYBR Green or TaqMan assays. Relative quantification was performed as described. ${ }^{23}$

Microarrays. Lung total RNA $(0.5-2 \mu \mathrm{g})$ was processed on Affymetrix GeneChip Mouse Genome 430 2.0 Array (Santa Clara, CA) or Applied Biosystems Mouse Whole Genome Arrays (Foster City, CA). Microarrays were analyzed respectively using the Robust Multichip Average method and the Bioconductor Linear Models for Microarray Data package and using the NeONORM method as described. ${ }^{29,30}$ MIAME-compliant microarray data are available at http://mace.ihes.fr (3133411542) and www.ncbi.nlm.nih.gov/gds (GSE45369).

Laser microdissection. Whole lungs were fixed, embedded in OCT compound (Sakura, Nagano, Japan) and frozen as described. ${ }^{31}$ Sections $(15-20 \mu \mathrm{m})$ were stained with cresyl violet as manufacturer procedure (Ambion, Austin, TX). Using the LMD AS microscope (Leica, Wetzlar, Germany), >10 samples of epithelial layer of bronchi or alveolar regions per experiment were cut out and collected into RNA lysis buffer.

Cell Culture. The normal human bronchial epithelial cell BEAS-2B was grown in complete epithelial cell culture medium (Promocell GmbH, Heidelberg, Germany). The human bronchial epithelial cell $16 \mathrm{HBE}$ was grown in Dulbecco's modified Eagle's medium (DMEM) with $5 \%$ fetal calf serum (FCS). The human type II pneumocyte cell A549 was grown in DMEM/F12 supplemented with 10\% FCS, 10 mM HEPES, 1 mM sodium pyruvate, nonessential amino acids. The Caco-2 human colon adenocarcinoma cell was grown in DMEM with $10 \%$ FCS, $10 \mathrm{~mm}$ HEPES, and non-essential amino acids. Cells were stimulated with $1 \mu \mathrm{g} \mathrm{ml}^{-1}$ flagellin or 1/10th dilution of BAL from vaccinated mice.

Statistical analysis. Statistical differences were analyzed using the Mann-Whitney test and were considered to be significant for $P$-values $<0.05$.
SUPPLEMENTARY MATERIAL is linked to the online version of the paper at http://www.nature.com/mi

\section{ACKNOWLEDGEMENTS}

We thank Dr Christophe Carnoy for critical reading of the manuscript, Dr Edward Miao for plasmids encoding NLRC4-deficient flagellins, and Dr Philippe Gosset for BEAS-2B and 16HBE cells. L.V.M., D.C., D.F., S.C., J.T. and J.C.S. were funded by INSERM, Institut Pasteur de Lille, Région Nord Pas de Calais (ARCir Europe ${ }^{\circ}$ R07028EE and PhD fellowship), Univ Lille Nord de France, Agence Nationale de Recherche sur le SIDA ( ${ }^{\circ} 2008$ / 058), INSERM-CONICET joint project ( $\left.\mathrm{n}^{\circ} 174\right)$, Réseau National des Génopoles ( $\mathrm{n}^{\circ}$ 203), and European Community (STREP SavinMucoPath INCO-CT-2006-032296). AGB was funded by the Agence Nationale pour la Recherche (Physio2007 n 013-01) and the Genopole Evry.

\section{DISCLOSURE}

The authors declared no conflict of interest.

c 2014 Society for Mucosal Immunology

\section{REFERENCES}

1. Mbow, M.L., De Gregorio, E, Valiante, N.M. \& Rappuoli, R. New adjuvants for human vaccines. Curr Opin Immunol 22, 411-416 (2010).

2. Marichal, T. et al. DNA released from dying host cells mediates aluminum adjuvant activity. Nat Med 17, 996-1002 (2011).

3. Beutler, B. et al. Genetic analysis of host resistance: Toll-like receptor signaling and immunity at large. Annu Rev Immunol 24, 353-389 (2006).

4. Kufer, T.A. \& Sansonetti, P.J. NLR functions beyond pathogen recognition. Nat Immunol 12, 121-128 (2011).

5. Moresco, E.M., LaVine, D \& Beutler, B. Toll-like receptors. Curr Biol 21, R488-R493 (2011).

6. Abreu, M.T. Toll-like receptor signalling in the intestinal epithelium: how bacterial recognition shapes intestinal function. Nat Rev Immunol 10, 131-144 (2010).

7. Helft, J, Ginhoux, F, Bogunovic, M \& Merad, M. Origin and functional heterogeneity of non-lymphoid tissue dendritic cells in mice. Immunol Rev 234, 55-75 (2010).

8. Sporri, R. Reis e Sousa C. Inflammatory mediators are insufficient for full dendritic cell activation and promote expansion of CD4 + Tcell populations lacking helper function. Nat Immunol 6, 163-170 (2005).

9. Sansonetti, P.J. War and peace at mucosal surfaces. Nat Rev Immunol 4, 953-964 (2004).

10. Lycke, N. Recent progress in mucosal vaccine development: potential and limitations. Nat Rev Immunol 12, 592-605 (2012).

11. Woodrow, K.A., Bennett, K.M. \& Lo, D.D. Mucosal vaccine design and delivery. Annu Rev Biomed Eng 14, 17-46 (2012).

12. Ramos, H.C., Rumbo, M \& Sirard, J.C. Bacterial flagellins: mediators of pathogenicity and host immune responses in mucosa. Trends Microbiol 12, 509-517 (2004).

13. Lamkanfi, M \& Dixit, V.M. Inflammasomes and their roles in health and disease. Annu Rev Cell Dev Biol 28, 137-161 (2012).

14. Sanders, C.J., Yu, Y, Moore, D.A. 3rd, Williams, I.R. \& Gewirtz, A.T. Humoral immune response to flagellin requires $T$ cells and activation of innate immunity. J Immunol 177, 2810-2818 (2006).

15. Didierlaurent, A. et al. Flagellin promotes myeloid differentiation factor 88-dependent development of Th2-type response. J Immunol 172, 6922-6930 (2004)

16. McSorley, S.J., Ehst, B.D., Yu, Y \& Gewirtz, A.T. Bacterial flagellin is an effective adjuvant for CD4(+) T cells in vivo. J Immunol 169, 3914-3919 (2002).

17. Vijay-Kumar, M, Carvalho, F.A., Aitken, J.D., Fifadara, N.H. \& Gewirtz, A.T. TLR5 or NLRC4 is necessary and sufficient for promotion of humoral immunity by flagellin. Eur J Immunol 40, 3528-3534 (2010).

18. Nempont, C, Cayet, D, Rumbo, M, Bompard, C, Villeret, V \& Sirard, J.C. Deletion of flagellin's hypervariable region abrogates antibody-mediated neutralization and systemic activation of TLR5-dependent immunity. J Immunol 181, 2036-2043 (2008). 
19. Wilson, R.H. et al. The Toll-like receptor 5 ligand flagellin promotes asthma by priming allergic responses to indoor allergens. Nat Med 18, 1705-1710 (2012).

20. Honko, A.N., Sriranganathan, N, Lees, C.J. \& Mizel, S.B. Flagellin is an effective adjuvant for immunization against lethal respiratory challenge with Yersinia pestis. Infect Immun 74, 1113-1120 (2006).

21. Pino, O, Martin, M \& Michalek, S.M. Cellular mechanisms of the adjuvant activity of the flagellin component FljB of Salmonella enterica Serovar Typhimurium to potentiate mucosal and systemic responses. Infect Immun 73, 6763-6770 (2005).

22. Uematsu, S. et al. Regulation of humoral and cellular gut immunity by lamina propria dendritic cells expressing Toll-like receptor 5. Nat Immunol 9, 769-776 (2008).

23. Van Maele, L. et al. TLR5 signaling stimulates the innate production of IL-17 and IL-22 by CD3(neg)CD127 + immune cells in spleen and mucosa. J Immunol 185, 1177-1185 (2010).

24. Kinnebrew, M.A. et al. Interleukin 23 production by intestinal CD103(+) $\mathrm{CD} 11 \mathrm{~b}(+)$ dendritic cells in response to bacterial flagellin enhances mucosal innate immune defense. Immunity 36, 276-287 (2012).

25. Sierro, F, Dubois, B, Coste, A, Kaiserlian, D, Kraehenbuhl, J.P. \& Sirard, J.C. Flagellin stimulation of intestinal epithelial cells triggers CCL20-mediated migration of dendritic cells. Proc Natl Acad Sci USA 98, 13722-13727 (2001).

26. Gewirtz, A.T. et al. Salmonella typhimurium translocates flagellin across intestinal epithelia, inducing a proinflammatory response. J Clin Invest 107, 99-109 (2001).

27. Fritz, J.H., Le Bourhis, L, Magalhaes, J.G. \& Philpott, D.J. Innate immune recognition at the epithelial barrier drives adaptive immunity: APCs take the back seat. Trends Immunol 29, 41-49 (2008).

28. Janot, L. et al. Radioresistant cells expressing TLR5 control the respiratory epithelium's innate immune responses to flagellin. Eur J Immunol 39, 1587-1596 (2009).

29. Eilebrecht, S, Pellay, F.X., Odenwalder, P, Brysbaert, G, Benecke, B.J. \& Benecke, A. EBER2 RNA-induced transcriptome changes identify cellular processes likely targeted during Epstein Barr Virus infection. BMC Res Notes 1, 100 (2008).

30. Noth, S, Brysbaert, G, Pellay, F.X. \& Benecke, A. High-sensitivity transcriptome data structure and implications for analysis and biologic interpretation. Genomics Proteomics Bioinformatics 4, 212-229 (2006).

31. Anderle, P. et al. Novel markers of the human follicle-associated epithelium identified by genomic profiling and microdissection. Gastroenterol 129, 321-327 (2005).

32. Mizel, S.B. \& Bates, J.T. Flagellin as an adjuvant: cellular mechanisms and potential. J Immunol 185, 5677-5682 (2010).

33. Miao, E.A. et al. Innate immune detection of the type III secretion apparatus through the NLRC4 inflammasome. Proc Natl Acad Sci USA 107, 3076-3080 (2010).

34. Lopez-Boado, Y.S., Espinola, M, Bahr, S \& Belaaouaj, A. Neutrophil serine proteinases cleave bacterial flagellin, abrogating its host responseinducing activity. J Immunol 172, 509-515 (2004).

35. Vonderviszt, F, Uedaira, H, Kidokoro, S \& Namba, K. Structural organization of flagellin. J Mol Biol 214, 97-104 (1990).

36. Le Borgne, M. et al. Dendritic cells rapidly recruited into epithelial tissues via CCR6/CCL20 are responsible for CD8(+) T cell crosspriming in vivo. Immunity 24, 191-201 (2006).

37. Eckmann, L, Kagnoff, M.F. \& Fierer, J. Epithelial cells secrete the chemokine interleukin-8 in response to bacterial entry. Infect Immun 61, 4569-4574 (1993).

38. McCormick, B.A., Colgan, S.P., Delp-Archer, C, Miller, S.I. \& Madara, J.L. Salmonella typhimurium attachment to human intestinal epithelial monolayers: transcellular signalling to subepithelial neutrophils. J Cell Biol 123, 895-907 (1993).
39. Evans, S.E., Xu, Y, Tuvim, M.J. \& Dickey, B.F. Inducible innate resistance of lung epithelium to infection. Annu Rev Physiol 72, 413-435 (2010).

40. Feuillet, $V$. et al. Involvement of Toll-like receptor 5 in the recognition of flagellated bacteria. Proc Natl Acad Sci USA 103, 12487-12492 (2006).

41. Hammad, H, Chieppa, M, Perros, F, Willart, M.A., Germain, R.N. \& Lambrecht, B.N. House dust mite allergen induces asthma via Toll-like receptor 4 triggering of airway structural cells. Nat Med 15, 410-416 (2009).

42. Nenci, A. et al. Epithelial NEMO links innate immunity to chronic intestinal inflammation. Nature 446, 557-561 (2007).

43. Didierlaurent, A. et al. Sustained desensitization to bacterial Toll-like receptor ligands after resolution of respiratory influenza infection. J Exp Med 205, 323-329 (2008).

44. Sato, A \& Iwasaki, A. Induction of antiviral immunity requires Toll-like receptor signaling in both stromal and dendritic cell compartments. Proc Natl Acad Sci USA 101, 16274-16279 (2004).

45. Hou, B, Reizis, B \& DeFranco, A.L. Toll-like receptors activate innate and adaptive immunity by using dendritic cell-intrinsic and -extrinsic mechanisms. Immunity 29, 272-282 (2008).

46. Lee, H.C. \& Ziegler, S.F. Inducible expression of the proallergic cytokine thymic stromal lymphopoietin in airway epithelial cells is controlled by NFkappaB. Proc Natl Acad Sci USA 104, 914-919 (2007).

47. Shang, L. et al. Toll-like receptor signaling in small intestinal epithelium promotes B-cell recruitment and IgA production in lamina propria. Gastroenterol 135, 529-538 (2008).

48. Pichavant, M. et al. Asthmatic bronchial epithelium activated by the proteolytic allergen Der $\mathrm{p} 1$ increases selective dendritic cell recruitment. $J$ Allergy Clin Immunol 115, 771-778 (2005).

49. Greter, M. et al. GM-CSF controls nonlymphoid tissue dendritic cell homeostasis but is dispensable for the differentiation of inflammatory dendritic cells. Immunity 36, 1031-1046 (2012).

50. Nolte, M.A., Leibundgut-Landmann, S, Joffre, O \& Reis e Sousa, C. Dendritic cell quiescence during systemic inflammation driven by LPS stimulation of radioresistant cells in vivo. J Exp Med 204, 1487-1501 (2007).

51. Murray, P.J. \& Smale, S.T. Restraint of inflammatory signaling by interdependent strata of negative regulatory pathways. Nat Immunol 13, 916-924 (2012).

52. Boone, D.L. et al. The ubiquitin-modifying enzyme A20 is required for termination of Toll-like receptor responses. Nat Immunol 5, 1052-1060 (2004).

53. Oshima, N. et al. A20 is an early responding negative regulator of Toll-like receptor 5 signalling in intestinal epithelial cells during inflammation. Clin Exp Immunol 159, 185-198 (2010).

54. Vereecke, L. et al. Enterocyte-specific A20 deficiency sensitizes to tumor necrosis factor-induced toxicity and experimental colitis. J Exp Med 207, 1513-1523 (2010).

55. Gilchrist, M. et al. Activating transcription factor 3 is a negative regulator of allergic pulmonary inflammation. J Exp Med 205, 2349-2357 (2008).

56. Taylor, G.A. et al. A pathogenetic role for TNF alpha in the syndrome of cachexia, arthritis, and autoimmunity resulting from tristetraprolin (TTP) deficiency. Immunity 4, 445-454 (1996).

57. Shaykhiev, R, Behr, J \& Bals, R. Microbial patterns signaling via Toll-like receptors 2 and 5 contribute to epithelial repair, growth and survival. PLOS One 3, e1393 (2008).

58. Burdelya, L.G. et al. An agonist of toll-like receptor 5 has radioprotective activity in mouse and primate models. Science 320, 226-230 (2008).

59. Munoz, N, Van Maele, L, Marques, J.M., Rial, A, Sirard, J.C. \& Chabalgoity, J.A. Mucosal administration of flagellin protects mice from Streptococcus pneumoniae lung infection. Infect Immun 78, 4226-4233 (2010). 